\title{
Decomposing the Garner interference paradigm: Evidence for dissociations between macrolevel and microlevel performance
}

\author{
BenJAMin J. Dyson \\ Ryerson University, Toronto, Ontario, Canada \\ AND \\ Philip T. Quinlan \\ University of York, York, England
}

\begin{abstract}
Three Garner interference experiments are described in which baseline, filtering, and correlated performance were assessed at both a macrolevel (condition average) and microlevel (intertrial contingency), using the pairwise combinations of auditory pitch, loudness, and location. Discrepancies between pairs of dimensions were revealed between macro- and microlevel estimates of performance and, also, between filtering costs and correlated benefits, relative to baseline. The examination of the intertrial effects associated with filtering costs suggested that effects of increased stimulus uncertainty were mandatory, whereas effects of irrelevant variation were not. The examination of the intertrial effects associated with correlated benefits suggested that the detection of stimulus repetition took precedence over that of stimulus change. Violations of standard horse race accounts of processing did not appear to stem from differences in the absolute or relative speeds of processing between dimensions but, rather, from the special role that certain dimensions (e.g., pitch) may play in certain modalities (e.g., audition). The utility of examining repetition effects is demonstrated by revealing a level of understanding regarding stimulus processing typically hidden by aggregated measures of performance.
\end{abstract}

A central use of the Garner $(1974,1976)$ interference paradigm is to establish the nature of interactions between properties of multidimensional stimuli by instructing participants to classify distinctive features from a single dimension over a number of different conditions. At the two extremes, stimuli may have a dimensional structure that is directly perceived (i.e., stimuli comprising separable or independent dimensions) or perceived in a unitary or holistic fashion in which one dimension cannot be considered without the other (i.e., stimuli comprising integral or interdependent dimensions). In the visual modality, size and diameter are thought to be separable dimensions of a circle (Garner \& Felfoldy, 1970), whereas height and width are thought to be integral dimensions of a rectangle (Felfoldy, 1974). Establishing the nature of dimensional interactions in perceptual classification tasks has repercussions for other central cognitive issues, such as feature binding (Treisman \& Gelade, 1980). Stimuli initially represented by separable dimensions require additional processing to combine those features into an object-based representation. Stimuli possessive of integral dimensions are primarily available as a unitary percept and require further processing to retrieve individual components (although see Dyson \& Quinlan, 2004, for a discussion of the potential differences between early- and late-object representation).
In terms of exposing the basic logic of the following experiments, a total of 4 stimuli are generated, constituting the orthogonal combination of two levels of Dimension $\mathrm{A}$ $\left(\mathrm{A}_{0}\right.$ and $\left.\mathrm{A}_{1}\right)$ and two levels of Dimension $\mathrm{B}\left(\mathrm{B}_{0}\right.$ and $\left.\mathrm{B}_{1}\right)$. In order to establish speed and accuracy of discriminability within each dimension, performance is initially examined in baseline conditions, where only values along the relevant dimension are allowed to vary. If the relevant dimension is $\mathrm{A}$, the stimuli can be either $\mathrm{A}_{0} \mathrm{~B}_{0}$ and $\mathrm{A}_{1} \mathrm{~B}_{0}$ or $\mathrm{A}_{0} \mathrm{~B}_{1}$ and $A_{1} B_{1}$. If the relevant dimension is $B$, the stimuli can be either $\mathrm{A}_{0} \mathrm{~B}_{0}$ and $\mathrm{A}_{0} \mathrm{~B}_{1}$ or $\mathrm{A}_{1} \mathrm{~B}_{0}$ and $\mathrm{A}_{1} \mathrm{~B}_{1}$. Converging evidence for the nature of dimensional interaction is then collected by comparing baseline performance against two additional conditions: a filtering task in which dimensional integrality should hinder processing and a correlated task in which dimensional integrality should help processing. In filtering conditions, values on both relevant and irrelevant dimensions change randomly over trials, but participants respond on the basis of a single relevant dimension. For instance, one response is associated with $\mathrm{A}_{0}$ and a different response is associated with $A_{1}$, and over trials, the stimuli $A_{0} B_{0}, A_{0} B_{1}, A_{1} B_{0}$, and $A_{1} B_{1}$ are used. If $A$ and $B$ are integral stimulus dimensions, it is predicted that the close binding of relevant and irrelevant information will lead to slower reaction times (RTs) and/or larger error rates in the filtering

B. J. Dyson, ben.dyson@psych.ryerson.ca 
condition, relative to the baseline condition (known as a filtering cost). In correlated conditions, values on both the relevant and irrelevant dimensions change, but the pairings of the relevant and irrelevant values remain constant. Regardless of whether $\mathrm{A}$ or $\mathrm{B}$ is the relevant dimension, stimuli in one correlated condition are $\mathrm{A}_{0} \mathrm{~B}_{0}$ and $\mathrm{A}_{1} \mathrm{~B}_{1}$, whereas in the other correlated condition, they are $\mathrm{A}_{0} \mathrm{~B}_{1}$ and $\mathrm{A}_{1} \mathrm{~B}_{0}$. Whereas the failure of selective attention associated with integral stimulus dimensions disrupts performance in the filtering condition, the same relationship should facilitate performance in the correlated conditions and, hence, lead to shorter RTs and/or lower error rates, relative to the baseline condition (known as a correlated benefit).

Filtering costs and correlated benefits continue to serve as important tools in the evaluation of dimensional interaction (see Grau \& Kemler Nelson, 1988, for other metrics), despite a number of concerns raised by the previous literature. First, although filtering costs and correlated benefits may be considered as different expressions of the same failure of selective attention to one of a pair of integral dimensions, these effects often dissociate from one another, leading to speculation that they may represent different forms of processing (e.g., Boenke, Ohl, Nikolaev, Lachmann, \& van Leeuwen, 2009; Patching \& Quinlan, 2002; van Leeuwen \& Bakker, 1995). Second, the occurrence of filtering costs and/or correlated benefits does not necessarily reveal the level at which the stimulus dimensions interact. Indeed, Pomerantz (1983) has identified multiple loci for cross talk during Garner classification, including perceptual, decisional, and response levels (see also Boenke et al., 2009; Caclin, McAdams, Smith, \& Giard, 2008; Melara \& Mounts, 1993, 1994; van Leeuwen \& Bakker, 1995). A third limitation explored in the present article is the idea that condition-average comparisons mask a number of independent trial-to-trial contributions to filtering costs and correlated benefits. Some of these may not address dimensional interaction per se but may, instead, highlight problems in the design of the Garner interference paradigm in general (Felfoldy, 1974; Huettel \& Lockhead, 1999; Shechter \& Hochstein, 1992). In order to proceed, we need to discuss in some detail the contingencies between the immediately previous $(n-1)$ and the current $(n)$ trial within all three conditions (see Table 1 for a summary).

Performance in the baseline condition reflects variation on the relevant dimension when either of a pair of stimuli is classified (e.g., Dimension A is relevant: $\mathrm{A}_{0} \mathrm{~B}_{0}$ and $\mathrm{A}_{1} \mathrm{~B}_{0}$ ), and as such, stimulus presentation can vary across trials in one of two ways. First, the same stimulus on trial $n-1$ may be re-presented on trial $n$ (e.g., $\mathrm{A}_{0} \mathrm{~B}_{0}$ followed by $\mathrm{A}_{0} \mathrm{~B}_{0}$ ), such that both values on both relevant

Table 1

Intertrial Contingencies Available Within the Three Condition Types of the Garner Interference Paradigm

\begin{tabular}{lccc}
\hline & Baseline & Filtering & Correlated \\
\hline Relevant repeat + irrelevant repeat & Yes & Yes & Yes \\
Relevant repeat + irrelevant change & No & Yes & No \\
Relevant change + irrelevant repeat & Yes & Yes & No \\
Relevant change + irrelevant change & No & Yes & Yes \\
\hline
\end{tabular}

and irrelevant dimensions are repeated (hereafter, RR, taking the order of relevant then irrelevant dimensional value repeating $[\mathrm{R}]$ or changing $[\mathrm{C}])$. Second, different stimuli may be presented across trials (e.g., $\mathrm{A}_{0} \mathrm{~B}_{0}$ followed by $\mathrm{A}_{1} \mathrm{~B}_{0}$ ), in which the relevant dimension changes but the irrelevant dimension is maintained (hereafter, CR). For the filtering condition employing orthogonal relevant and irrelevant variation between four stimuli (e.g., $\mathrm{A}_{0} \mathrm{~B}_{0}$, $A_{0} B_{1}, A_{1} B_{0}, A_{1} B_{1}$ ), stimulus presentation varies across trials in four different ways. These include the same intertrial contingencies found in baseline conditions (RR, $\mathrm{CR}$ ), in addition to two new contingencies as a result of the introduction of irrelevant variation: $\mathrm{RC}$ and $\mathrm{CC}$ trials. For the correlated condition utilizing synchronized relevant and irrelevant variation (e.g., $\mathrm{A}_{0} \mathrm{~B}_{0}$ and $\mathrm{A}_{1} \mathrm{~B}_{1}$ ), stimulus presentation varies in that both the relevant and irrelevant dimensions are repeated (RR), or completely different stimuli are presented across trials, such that both the relevant and irrelevant dimensions change (CC).

Traditionally, performance in such Garner tasks has been studied at the level of condition averages (something we refer to here as the macrolevel). However, more fine-grained microlevel analyses are possible. It is possible to decompose the macrolevel filtering cost and the correlated benefit into their constituent microlevel parts; Estimator 1 represents the traditional calculation of filtering cost unpacked with respect to trial-to-trial transitions between filtering and baseline conditions:

$$
\begin{aligned}
& \{[\mathrm{E}(\mathrm{RT} \mid \mathrm{RR} \text {; filtering })+\mathrm{E}(\mathrm{RT} \mid \mathrm{RC} \text {; filtering }) \\
& +\mathrm{E}(\mathrm{RT} \mid \mathrm{CR} \text {; filtering })+\mathrm{E}(\mathrm{RT} \mid \mathrm{CC} \text {; filtering })] / 4\} \\
& -\{[\mathrm{E}(\mathrm{RT} \mid \mathrm{RR} \text {; baseline })+\mathrm{E}(\mathrm{RT} \mid \mathrm{CR} \text {; baseline })] / 2\}
\end{aligned}
$$

Viewed in this way, one clear contribution to the filtering cost is the addition of two intertrial contingencies that represent the presence of irrelevant variation (i.e., $\mathrm{RC}$ and CC; Huettel \& Lockhead, 1999; Pomerantz, 1983; Shechter \& Hochstein, 1992) that are not present in the baseline condition. However, these intertrial contingencies also bring about an increase in stimulus uncertainty (Ben-Artzi \& Marks, 1995, 1999): a larger number of stimulus tokens in the filtering condition (i.e., 4) relative to the baseline condition (i.e., 2). Although traditional condition-average performance cannot be used to separate out the contributions of irrelevant variation and stimulus uncertainty on any putative filtering cost, the recalculation of filtering costs according to intertrial contingency can. Estimator 2 provides a measure of irrelevant variation by subtracting from the grand average of the filtering condition those intertrial contingencies that do not represent change in an irrelevant dimension. In this way, the effect of irrelevant variation is maximized, whereas the effect of stimulus uncertainty is minimized, since the calculation is performed within condition:

$$
\begin{aligned}
& \{[\mathrm{E}(\mathrm{RT} \mid \mathrm{RR} \text {; filtering })+\mathrm{E}(\mathrm{RT} \mid \mathrm{RC} \text {; filtering }) \\
& +\mathrm{E}(\mathrm{RT} \mid \mathrm{CR} \text {; filtering })+\mathrm{E}(\mathrm{RT} \mid \mathrm{CC} \text {; filtering })] / 4\} \\
& -\{[\mathrm{E}(\mathrm{RT} \mid \mathrm{RR} \text {; filtering })+\mathrm{E}(\mathrm{RT} \mid \mathrm{CR} \text {; filtering })] / 2\} .
\end{aligned}
$$

An additional estimator is also available that allows us to examine only those intertrial contingencies that are 
shared between filtering and baseline conditions, by comparing contingencies between conditions in the absence of irrelevant variation. Estimator 3 therefore provides a measure of stimulus uncertainty:

$$
\begin{aligned}
& \{[\mathrm{E}(\mathrm{RT} \mid \mathrm{RR} \text {; filtering })+\mathrm{E}(\mathrm{RT} \mid \mathrm{CR} \text {; filtering })] / 2\} \\
& -\{[\mathrm{E}(\mathrm{RT} \mid \mathrm{RR} ; \text { baseline })+\mathrm{E}(\mathrm{RT} \mid \mathrm{CR} \text {; baseline })] / 2\} .
\end{aligned}
$$

Taken collectively, consideration of microlevel performance identifies two independent contributions to the macrolevel - namely, irrelevant variation and stimulus uncertainty - that cumulatively account for any overall filtering cost, given that

$$
\text { (1) }=(2)+(3) \text {. }
$$

Not only does this provide us with hitherto unavailable information regarding finer-grained performance during Garner interference, but also it allows us to examine two effects of potentially different theoretical importance. Arguably, it is only an effect of irrelevant variation that implicates the eventual retrieval of task-irrelevant information, consistent with the definition of dimensional integrality. In contrast, an effect of stimulus uncertainty does not necessarily invoke the explicit representation of dimensional or featural information but merely demands increased processing variation (Lockhead, 1972).

The evaluation of correlated benefits may also be unpacked in a similar way, leading to the identification of two independent microlevel contributions that cumulatively account for any macrolevel effect observed. Estimator 5 represents the traditional calculation of correlated benefits unpacked with respect to trial-to-trial transitions between correlated and baseline conditions:

$$
\begin{aligned}
& \{[\mathrm{E}(\mathrm{RT} \mid \mathrm{RR} ; \text { correlated })+\mathrm{E}(\mathrm{RT} \mid \mathrm{CC} ; \text { correlated })] / 2\} \\
& -\{[\mathrm{E}(\mathrm{RT} \mid \mathrm{RR} ; \text { baseline })+\mathrm{E}(\mathrm{RT} \mid \mathrm{CR} ; \text { baseline })] / 2\} .
\end{aligned}
$$

Traditional accounts of correlated benefit appeal to the idea of redundancy gain in that, for pairs of integral dimensions under conditions of correlated presentation, the value of the first dimension completely predicts the value of the second dimension: There are only cases of complete stimulus repetition and complete stimulus change. This contrasts with baseline presentation in that the value of the relevant dimension is orthogonal to the (fixed) value of the irrelevant dimension, leading to cases of complete stimulus repetition and cases of relevant change only. As was noted by Felfoldy (1974), identical RR contingencies apparent in both correlated and baseline conditions reflect complete stimulus repetition across two dimensions, although it should only be in the correlated condition that there is an advantage attributable to redundancy gain. Therefore, a comparison of complete stimulus repetition trials across correlated and baseline conditions, as shown in Estimator 6, indicates whether dimensional value redundancy is apparent during complete stimulus and response repetition (hereafter, redundancy repetition $[\mathrm{R}])$ :

$\{\mathrm{E}(\mathrm{RT} \mid \mathrm{RR}$; correlated $)-\mathrm{E}(\mathrm{RT} \mid \mathrm{RR} ;$ baseline $)\} / 2$.
The correlated condition is also unique in providing an example of dimensional value redundancy on CC trials. This is in contrast to the baseline condition, in which response change is marked only by relevant dimensional value change (CR trials). Estimator 7 addresses whether an additional change in the irrelevant dimension is critical to establishing processing facilitation in correlated conditions when response also changes (hereafter, redundancy change [C]):

$$
\{\mathrm{E}(\mathrm{RT} \mid \mathrm{CC} \text {; correlated })-\mathrm{E}(\mathrm{RT} \mid \mathrm{CR} \text {; baseline })\} / 2 \text {. }
$$

Estimators 6 and 7 partition macrolevel correlated benefits into independent microlevel effects of redundancy during repetition and change, given that

$$
(5)=(6)+(7) \text {. }
$$

As in the decomposition of filtering costs, a comparison between these two effects is also of theoretical interest, in that the availability of irrelevant information during baseline conditions can be assessed by comparing redundancy repetition with redundancy change. Specifically, if the value of the irrelevant dimension influences processing during baseline conditions, cases of baseline repetition are closer in nature to correlated repetition (RR; baseline vs. RR; correlated) than cases of baseline change are to correlated change (CR; baseline vs. CC; correlated). Consequently, Estimator 7 should yield more robust effects than Estimator 6.

A final estimator evaluates one aspect of the default nature of information processing via the initiation of the bypass rule (Kruger \& Shapiro, 1981). This rule states that processing is facilitated when both the stimulus and response are identical across consecutive trials and when both the stimulus and response are different across consecutive trials (Rabbitt, 1992). Intertrial contingencies in correlated conditions represent both expressions of this rule via $\mathrm{RR}$ and $\mathrm{CC}$ trials, respectively. Although either outcome allows for the curtailment of more detailed analysis and for the initiation of a responding shortcut (Pashler \& Baylis, 1991), it is not clear whether one form of bypass has priority over another. Consequently, the valence of the outcome of Estimator 9 will provide some insight in this regard:

$$
\{\mathrm{E}(\mathrm{RT} \mid \mathrm{RR} \text {; correlated })-\mathrm{E}(\mathrm{RT} \mid \mathrm{CC} \text {; correlated })\} .
$$

In summary, the present experimental series were motivated by four aims. The first was to examine filtering costs and correlated benefits at the macrolevel using average condition RTs in the traditional manner across a range of auditory classification tasks. Second, the joint contributions of irrelevant variation and stimulus uncertainty on any observed filtering cost were examined at a microlevel by comparing the stimulus relations across consecutive trials in the filtering and baseline conditions. Third, the consequences of redundancy repetition and change on any observed correlated benefit were also estimated at a microlevel by comparing the stimulus relations across consecutive trials in the correlated and baseline conditions. Fourth, the comparison of macrolevel and microlevel 
performance was performed across a variety of auditory dimensions to yield converging evidence for some of the different types of stimulus interactions available when sound is processed.

\section{EXPERIMENT 1}

Although concepts of integrality and separability have traditionally been reserved for visual dimensions (Kemler Nelson, 1993; Maddox, 1992), there are a number of studies that have reported the status of dimensional interaction within the auditory domain for a variety of sound dimensions, including pitch, harmonicity, timbre, location, loudness, and vocal quality (e.g., Caclin, Giard, Smith, \& McAdams, 2007; Caclin et al., 2008; Dyson \& Alain, 2008; Dyson \& Quinlan, 2004; Grau \& Kemler Nelson, 1988; Harris, Pikler, Hoffman, \& Ehmer, 1958; Krumhansl \& Iverson, 1992; Melara \& Marks, 1990; Mondor, Zatorre, \& Terrio, 1998; Patching \& Quinlan, 2002; Wood, 1975). The present aim was not to provide evidence that the nature of an interaction between any pair of auditory dimensions is one or the other but, rather, to detail how dimensional relations estimated at the macrolevel relate to estimates derived at the microlevel. With this caveat in mind, we shall tackle the pairwise interactions between pitch, loudness, and location over three separate experiments, starting with pitch and loudness.

In an extensive study by Grau and Kemler Nelson (1988), multidimensional auditory stimuli varying with respect to pitch and loudness were found to produce both filtering costs and correlated benefits. This, in addition to other behavioral criteria (see also Garner, 1974; Wood, 1975), provided strong evidence that these attributes were integral and, therefore, processed interdependently (see also Zmigrod \& Hommel, 2009). Similarly intimate relations between pitch and loudness were reported by Harris et al. (1958), who noted that changing the value of one dimension seemed to change the perception of the value of the other dimension: "A slight change in intensity seems to produce a pitch change, and vice versa, so that the attributes are not in any real sense separable" (pp. 234-235). The direction of this interaction was also noted by Grau and Kemler Nelson, who observed that an increase in loudness led to a perceived increase in pitch.

However, one factor that appears to influence the expression of separability or integrality is the speed with which dimensional values can be discriminated under baseline conditions (Melara \& Mounts, 1993). If the speeds with which the dimensions are processed are different at baseline, the data in the other classification tasks lend themselves to analysis by way of horse race accounts of processing (Mordkoff \& Yantis, 1991; Mordkoff, Yantis, \& Egeth, 1990). Here, general principles of processing exist for multidimensional stimuli ( $\mathrm{AB}$ ) when the values of one dimension (e.g., $\mathrm{A}_{0}$ and $\mathrm{A}_{1}$ ) are discriminated more quickly than the values of another dimension (e.g., $\mathrm{B}_{0}$ and $\mathrm{B}_{1}$ ). When the retrieval of Dimension $\mathrm{A}$ is required for task completion, task-irrelevant Dimension B will have a negligible effect on processing as a result of its slower discrimination speed. However, if Dimension B is task relevant, Dimension A will influence the processing of B, increasing the likelihood of both processing inhibition when both dimensions vary orthogonally (i.e., filtering costs) and processing facilitation when both dimensions vary in a redundant fashion (i.e., correlated benefits). This then allows for intermediate levels of dimensional interaction to present themselves, in so-called asymmetrical integral (interchangeable with asymmetrical separable) relationships (Garner, 1976; Pomerantz \& Sager, 1975). In Experiment 1, we set out to examine macro- and microlevel performance in a Garner interference paradigm utilizing a relatively fast pitch dimension and a relatively slow loudness dimension.

\section{Method}

Participants. All data collection was carried out at the University of Sussex. Informed consent for 16 participants was obtained prior to the experiment. Each individual took part in two separate 1-h sessions. The sample consisted of 13 females and 14 right-handers, with ages ranging from 19 to 28 years $(M=20.19, S D=2.40)$. All volunteers reported normal hearing.

Stimuli and Apparatus. Four tones of $100-\mathrm{msec}$ duration with 10 -msec linear onset/offset ramps were created using SoundEdit16 (Macromedia), using the orthogonal combinations of high $(1500 \mathrm{~Hz})$ and low $(250 \mathrm{~Hz})$ frequencies and soft (approximately $70 \mathrm{~dB}$ ) and loud (approximately $80 \mathrm{~dB}$ ) intensity values. As a result of the recommended 2-dB adjustment for subjective loudness between these specific tone frequencies (Woods, Alain, \& Ogawa, 1998), the high tone was calibrated binaurally at 80 and $70 \mathrm{~dB}$ for loud and soft intensities, respectively, whereas the low tone was calibrated binaurally at 82 and $72 \mathrm{~dB}$, respectively, using Sennheiser HD580 headphones, and an artificial ear and measurement amplifier (Brüel \& Kjær Type 2610). Stimulus presentation was controlled via PsyScope (Cohen, MacWhinney, Flatt, \& Provost, 1993) with RTs recorded using a PsyScope button box. All testing was carried out in a single-skinned sound-attenuating chamber.

Design. The participants completed two counterbalanced experimental sessions in which they were required to categorize singly presented tones with respect to either pitch (high or low) or loudness (soft or loud). Each session required the completion of five conditions - namely, first baseline, second baseline, positively correlated, negatively correlated, and filter. Table 1 provides a summary of intertrial contingencies available in each of the three condition types. The first-baseline and second-baseline conditions differentiated themselves according to which particular value of the irrelevant dimension was presented. The positively correlated (or congruent) and negatively correlated (or incongruent) conditions differentiated themselves on the basis of the particular stimuli selected for presentation (see Patching \& Quinlan, 2002, for a fuller discussion of these issues). Notions of congruency between loudness and pitch information were guided by previous research in which lower tones were paired with softer intensity and higher tones were paired with louder intensity (Grau \& Kemler Nelson, 1988; Harris et al., 1958; Woods et al., 1998). For both versions of the baseline and correlated conditions, the participants completed a practice block of 16 stimulus pairs and an experimental block of 160 stimulus pairs, and performance was aggregated across the different versions prior to analysis. In order to arrive at the same number of observations for each type of intertrial contingency within the filtering condition, relative to the other conditions, the set of 16 practice followed by 160 experimental trial pairs was repeated over four runs of the filtering condition for both pitch and loudness. The ordering of the total eight conditions for each judgment was randomized across participants. All intertrial contingencies appropriate to each condition were pre- 
Table 2

Macrolevel Mean Reaction Times (RTs) and Percentage Error Rates (Both With Standard Errors, $S E$ ) Across Experiments 1-3

\begin{tabular}{|c|c|c|c|c|}
\hline \multirow[b]{2}{*}{ Condition } & \multicolumn{2}{|c|}{ RT (msec) } & \multicolumn{2}{|c|}{ Error $(\%)$} \\
\hline & $M$ & $\overline{S E}$ & $M$ & $S E$ \\
\hline \multicolumn{5}{|c|}{ Experiment 1 (Pitch vs. Loudness) } \\
\hline \multicolumn{5}{|l|}{ Pitch } \\
\hline Baseline & 378 & 32 & 2.02 & 0.42 \\
\hline Filtering & 377 & 26 & 2.29 & 0.51 \\
\hline Correlated & 353 & 26 & 1.67 & 0.32 \\
\hline \multicolumn{5}{|l|}{ Loudness } \\
\hline Baseline & 416 & 22 & 4.55 & 1.49 \\
\hline Filtering & 507 & 24 & 6.22 & 0.89 \\
\hline Correlated & 376 & 23 & 2.31 & 2.32 \\
\hline \multicolumn{5}{|c|}{ Experiment 2 (Azimuth vs. Loudness) } \\
\hline \multicolumn{5}{|c|}{ Azimuth } \\
\hline Baseline & 272 & 10 & 2.07 & 0.39 \\
\hline Filtering & 276 & 8 & 1.71 & 0.37 \\
\hline Correlated & 275 & 11 & 2.07 & 0.60 \\
\hline \multicolumn{5}{|l|}{ Loudness } \\
\hline Baseline & 405 & 24 & 5.58 & 0.93 \\
\hline Filtering & 465 & 29 & 8.46 & 1.45 \\
\hline Correlated & 356 & 18 & 4.77 & 1.37 \\
\hline \multicolumn{5}{|c|}{ Experiment 3 (Azimuth vs. Pitch) } \\
\hline \multicolumn{5}{|l|}{ Azimuth } \\
\hline Baseline & 328 & 17 & 1.86 & 0.54 \\
\hline Filtering & 370 & 21 & 2.69 & 0.68 \\
\hline Correlated & 332 & 18 & 1.43 & 0.39 \\
\hline \multicolumn{5}{|l|}{ Pitch } \\
\hline Baseline & 387 & 20 & 2.30 & 0.38 \\
\hline Filtering & 425 & 21 & 2.12 & 0.67 \\
\hline Correlated & 379 & 21 & 1.97 & 0.46 \\
\hline
\end{tabular}

sented with equal probability, and consecutive trial relations were equally represented by all stimulus pairings.

Procedure. Each trial began with a black screen for $250 \mathrm{msec}$. This was followed by a white central fixation cross for $500 \mathrm{msec}$, after which an auditory stimulus was played over headphones. The participants were required to categorize the task-relevant dimension of the stimulus as quickly and accurately as possible, by pressing one of two buttons on a PsyScope button box assigned to the index finger of each hand. The hardware was vertically oriented during pitch responding, with participants using a top button to respond high and a bottom button to respond low, and also was vertically oriented during loudness responding, with participants using a top button to respond loud and a bottom button to respond soft. After a short response-checking period of $50 \mathrm{msec}$, visual feedback was provided center screen in the form of "correct" or "error" for $500 \mathrm{msec}$. The next trial began with the presentation of a black screen, thereby establishing a response-stimulus interval (RSI) of 1,300 msec.

Data analysis. For all the experiments, only the RT and error rate data from trial $n$ in the experimental blocks were considered in the analysis. RTs were included if and only if correct responses were given on trials $n-1$ and $n$. Errors were defined as a correct response given at trial $n-1$ followed by an incorrect response given at trial $n$ (after Quinlan, 1999), and individual error rates were arcsin transformed prior to analysis (Winer, 1962). RT outliers were removed according to the recursive procedure outlined in van Selst and Jolicœur (1994) and Jolicœur (1999). Across Experiments 1-3, the factor of dimension varied as a function of the attributes used (pitch and loudness for Experiment 1; azimuth and loudness for Experiment 2; azimuth and pitch for Experiment 3). To retrieve macrolevel performance, both RTs and error rates across Experiments 1-3 were submitted to separate $2 \times 3$ repeated measures ANOVAs containing the factors of dimension and condition (baseline, filtering, correlated). All interactions were further decomposed using Tukey's HSD test $(p<.05)$. In evaluating microlevel performance related to filtering costs and correlated benefits, RT estimates of irrelevant variation (Estimator 2) and stimulus uncertainty (3) in the filtering condition and of redundancy during repetition (6), redundancy during change (7), and bypass rule (9) in the correlated condition were submitted to paired-sample $t$ tests relative to the dimensions used in the experiment, in addition to comparing individual effects with zero.

\section{Results}

Macrolevel analysis. Table 2 provides summary statistics for the three main condition types in Experiment 1, and the results of the related ANOVAs can be found in Table 3 . The RT data revealed main effects of dimension $(p=.005)$, with pitch judgments generally enacted more quickly than loudness judgments. The main effect of condition ( $p<.001)$, together with a significant interaction between dimension and condition $(p<.001)$, revealed no significant differences across conditions during pitch judgment. In contrast, the filtering condition was significantly slower and the correlated condition was significantly faster than the baseline condition during loudness judgments. Error rate analyses replicated the RT

Table 3

Results of the Two-Way Repeated Measures Reaction Time and Error Rate ANOVAs Between Pairwise Attributes (Pitch, Loudness, Azimuth) and Condition (Baseline, Filtering, Correlated) Across Experiments 1-3

\begin{tabular}{|c|c|c|c|c|c|c|c|c|c|}
\hline \multirow[b]{2}{*}{ Factor } & \multirow[b]{2}{*}{$d f$} & \multicolumn{4}{|c|}{ Reaction Times } & \multicolumn{4}{|c|}{ Error Rates } \\
\hline & & $F$ & $M S_{\mathrm{e}}$ & $p$ & $\eta_{\mathrm{p}}^{2}$ & $F$ & $M S_{\mathrm{e}}$ & $p$ & $\eta_{\mathrm{p}}^{2}$ \\
\hline \multicolumn{10}{|c|}{ Experiment 1 (Pitch vs. Loudness) } \\
\hline Dimension (D) & 1,15 & 11.12 & 8,706 & .005 & .426 & 11.66 & .010 & .003 & .437 \\
\hline Condition (C) & 2,30 & 62.82 & 780 & $<.001$ & .807 & 10.02 & .004 & $<.001$ & .401 \\
\hline $\mathrm{D} \times \mathrm{C}$ & 2,30 & 20.21 & 1,337 & $<.001$ & .574 & 5.77 & .003 & .008 & .278 \\
\hline \multicolumn{10}{|c|}{ Experiment 2 (Azimuth vs. Loudness) } \\
\hline D & 1,15 & 58.20 & 7,402 & $<.001$ & .795 & 20.78 & .019 & $<.001$ & .581 \\
\hline $\mathrm{C}$ & 2,30 & 23.96 & 1,020 & $<.001$ & .615 & 5.66 & .004 & .008 & .274 \\
\hline $\mathrm{D} \times \mathrm{C}$ & 2,30 & 18.36 & 1,289 & $<.001$ & .550 & 5.41 & .006 & .010 & .265 \\
\hline \multicolumn{10}{|c|}{ Experiment 3 (Azimuth vs. Pitch) } \\
\hline $\mathrm{D}$ & 1,15 & 11.67 & 5,904 & .004 & .438 & 0.13 & .004 & .724 & .009 \\
\hline $\mathrm{C}$ & 2,30 & 21.95 & 815 & $<.001$ & .594 & 2.69 & .001 & .084 & .152 \\
\hline $\mathrm{D} \times \mathrm{C}$ & 2,30 & 0.27 & 1,075 & .768 & .017 & 1.84 & .001 & .176 & .109 \\
\hline
\end{tabular}


interaction ( $p=.008$ ), showing the absence of variation in error rates during pitch judgments, whereas error rates for loudness judgments were generally larger and particularly low under correlated conditions.

Microlevel analysis. Table 4 provides summary statistics for individual intertrial contingencies across all three conditions. Figure 1 provides a graphical representation of both filtering cost and correlated benefit effects, and the results of the relevant $t$ tests can be found in Table 5. The contribution of irrelevant variation to the overall filtering cost was present for both pitch $(5 \mathrm{msec}$; $p=.029)$ and loudness $(37 \mathrm{msec} ; p<.001)$ responding, although the magnitude of the effect was larger for loudness responding $(p=.001)$. Similarly, the estimate of stimulus uncertainty was larger $(p=.004)$ and departed significantly from zero $(p<.001)$ only for loudness responding $(54 \mathrm{msec})$, relative to pitch responding ( $-5 \mathrm{msec})$. Regarding correlated benefits, estimates of redundancy during repetition significantly departed from zero for both pitch and loudness responding [-16 msec $(p=.022)$ and $-24 \operatorname{msec}(p<.001)$, respectively], and these estimates were not significantly different from one another $(p=.291)$. Despite the fact that estimates of redundancy during change did not significantly differ from one another $(p=.347)$, loudness responding showed an effect significantly different from zero $(-17 \mathrm{msec} ; p=$ $.030)$, whereas pitch responding did not $(-9 \mathrm{msec} ; p=$ .067). Finally, preferences for complete stimulus repetition (RR) over complete stimulus change (CC) were observed during both pitch $[t(15)=4.04, p=.001]$ and loudness $[t(15)=4.63, p<.001]$ responding during correlated conditions.

\section{Discussion}

The examination of repetition effects in Experiment 1 provided a much more detailed account of the processes underlying dimensional interaction, relative to traditional analyses of the Garner paradigm. Upon a standard reading based on average condition performance, pitch and loudness show evidence of an asymmetrical separable re-

Table 4

Microlevel Mean Reaction Times (RTs; With Standard Errors) and Percentage Error Rates Across Experiments 1-3

\begin{tabular}{|c|c|c|c|c|c|c|c|c|c|}
\hline & \multicolumn{2}{|c|}{ RT (msec) } & \multicolumn{2}{|c|}{ Error $(\%)$} & & \multicolumn{2}{|c|}{ RT (msec) } & \multicolumn{2}{|c|}{ Error $(\%)$} \\
\hline & $M$ & $S E$ & $M$ & $S E$ & & $M$ & $S E$ & $M$ & $S E$ \\
\hline \multicolumn{5}{|c|}{ Experiment 1 (Pitch vs. Loudness) } & \multicolumn{5}{|c|}{ Experiment 2 (Continued) } \\
\hline Pitch & & & & & Loudness & & & & \\
\hline Baseline & & & & & Baseline & & & & \\
\hline RR & 369 & 34 & 1.96 & 0.70 & $\mathrm{RR}$ & 396 & 24 & 4.23 & 0.73 \\
\hline $\mathrm{CR}$ & 386 & 30 & 2.08 & 0.44 & $\mathrm{CR}$ & 414 & 25 & 6.94 & 1.36 \\
\hline Filtering & & & & & Filtering & & & & \\
\hline $\mathrm{RR}$ & 357 & 26 & 0.92 & 0.44 & $\mathrm{RR}$ & 464 & 29 & 2.67 & 0.59 \\
\hline $\mathrm{RC}$ & 378 & 26 & 2.60 & 0.65 & $\mathrm{RC}$ & 473 & 32 & 12.56 & 2.06 \\
\hline $\mathrm{CR}$ & 388 & 26 & 3.34 & 1.00 & $\mathrm{CR}$ & 464 & 31 & 11.73 & 2.09 \\
\hline $\mathrm{CC}$ & 387 & 26 & 2.94 & 0.57 & $\mathrm{CC}$ & 459 & 27 & 6.87 & 1.68 \\
\hline Correlated & & & & & Correlated & & & & \\
\hline RR & 338 & 25 & 1.59 & 0.38 & RR & 345 & 17 & 3.62 & 1.04 \\
\hline $\mathrm{CC}$ & 368 & 28 & 1.75 & 0.46 & $\mathrm{CC}$ & 366 & 21 & 5.92 & 1.79 \\
\hline Loudness & & & & & & iment & imut & Pitch) & \\
\hline Baseline & & & & & & การ & & & \\
\hline RR & 403 & 25 & 2.26 & 0.61 & $\begin{array}{l}\text { Azumuth } \\
\text { Baseline }\end{array}$ & & & & \\
\hline $\begin{array}{c}\mathrm{CR} \\
\text { Filtering }\end{array}$ & 429 & 19 & 6.84 & 2.60 & RR & 324 & 16 & 2.29 & 0.91 \\
\hline $\begin{array}{l}\text { Filtering } \\
\text { RR }\end{array}$ & 426 & 24 & 0.76 & 0.25 & CR & 331 & 18 & 1.49 & 0.35 \\
\hline $\mathrm{RC}$ & 564 & 26 & 10.44 & 1.60 & Filtering & & & & \\
\hline $\mathrm{CR}$ & 515 & 20 & 10.77 & 1.76 & RR & 343 & 20 & 0.72 & 0.31 \\
\hline $\mathrm{CC}$ & 524 & 33 & 2.90 & 0.81 & $\mathrm{RC}$ & 406 & 21 & 5.82 & 1.80 \\
\hline Correlated & & & & & $\mathrm{CR}$ & 368 & 24 & 3.52 & 0.84 \\
\hline $\mathrm{RR}$ & 355 & 22 & 1.58 & 0.61 & $\mathrm{CC}$ & 364 & 23 & 0.72 & 0.37 \\
\hline $\mathrm{CC}$ & 396 & 24 & 3.05 & 0.87 & Correlated & & & & \\
\hline \multirow{2}{*}{\multicolumn{5}{|c|}{ Experiment 2 (Azimuth vs. Loudness) }} & RR & 325 & 17 & 1.28 & 0.50 \\
\hline & & & & & $\mathrm{CC}$ & 340 & 20 & 1.57 & 0.51 \\
\hline Azimuth & & & & & Pitch & & & & \\
\hline Baseline & & & & & Baseline & & & & \\
\hline RR & 275 & 10 & 2.12 & 0.43 & RR & 374 & 19 & 1.82 & 0.40 \\
\hline $\mathrm{CR}$ & 269 & 9 & 2.02 & 0.47 & $\mathrm{CR}$ & 399 & 21 & 2.79 & 0.63 \\
\hline Filtering & & & & & Filtering & & & & \\
\hline RR & 275 & 9 & 2.00 & 0.56 & RR & 394 & 21 & 0.91 & 0.43 \\
\hline $\mathrm{RC}$ & 280 & 9 & 2.16 & 0.68 & $\mathrm{RC}$ & 437 & 23 & 4.07 & 0.90 \\
\hline $\mathrm{CR}$ & 276 & 8 & 1.59 & 0.46 & $\mathrm{CR}$ & 438 & 21 & 1.83 & 0.38 \\
\hline $\mathrm{CC}$ & 273 & 8 & 1.11 & 0.41 & CC & 429 & 22 & 1.67 & 0.30 \\
\hline Correlated & & & & & Correlated & & & & \\
\hline RR & 279 & 12 & 2.13 & 0.72 & RR & 360 & 20 & 1.12 & 0.37 \\
\hline $\mathrm{CC}$ & 271 & 11 & 2.02 & 0.59 & $\mathrm{CC}$ & 399 & 23 & 2.81 & 0.73 \\
\hline
\end{tabular}

Note-RR, relevant repeat-irrelevant repeat; RC, relevant repeat-irrelevant change; CR, relevant change-irrelevant repeat; CC, relevant change-irrelevant change. 
A

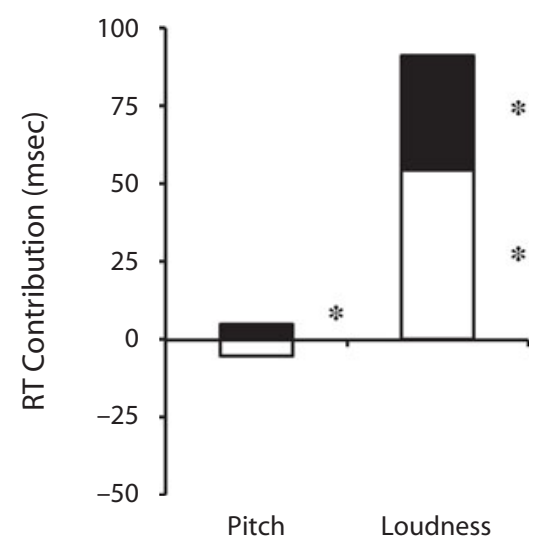

B

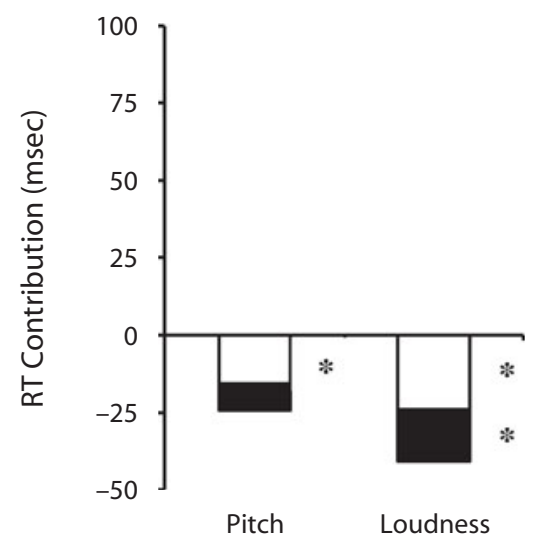

Filtering Costs

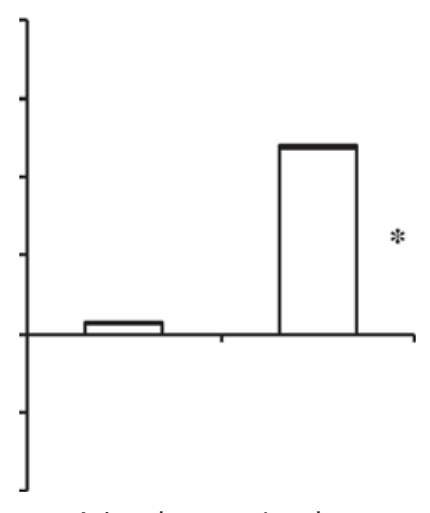

Azimuth Loudness

\section{Correlated Benefits}

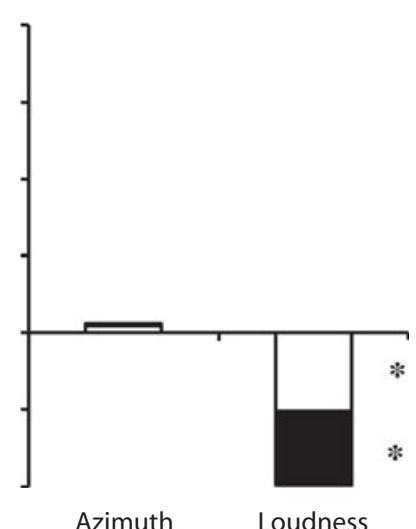

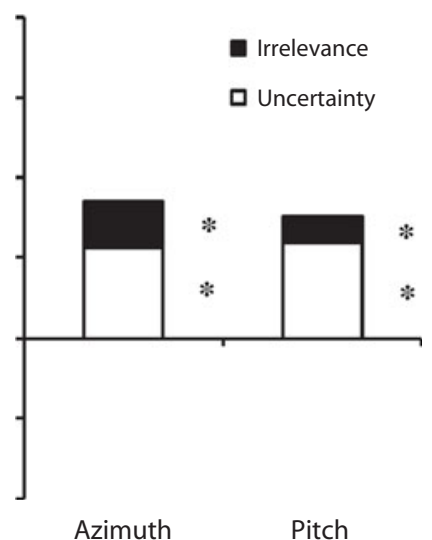

Azimuth Pitch

Figure 1. (A) Microlevel filtering costs (irrelevant variation, stimulus uncertainty) and (B) microlevel correlated benefits (redundancy R, redundancy C) in Experiment 1 using the auditory dimensions of pitch and loudness, Experiment 2 using the auditory dimensions of azimuth and loudness, and Experiment 3 using the auditory dimensions of azimuth and pitch. The sum of the two microlevel effects constitutes the macrolevel cost or benefit as retrieved from condition-average responding. Asterisks indicate that individual effects are different from zero at $p<.05$. RT, reaction time.

lationship, similar to the relationship shown between the auditory dimensions of pitch and harmonicity in previous auditory studies (Dyson \& Alain, 2008). Consistent with horse race accounts of processing (Mordkoff et al., 1990), when differences exist in baseline discriminability between dimensions, such that judgments about one dimension are faster than judgments about another, the stimulus should act as integral when the slower dimension is task relevant but as separable when the faster dimension is task relevant (Grau \& Kemler Nelson, 1988; Harris et al., 1958; Woods et al., 1998). This is exactly what was found at the macrolevel (as reflected in condition average performance): During loudness judgment, additional information regarding pitch facilitated processing during correlated conditions and hindered processing during filtering conditions, but during pitch responding, additional variation in loudness neither helped nor hindered processing to a significant degree.

Yet, as we have already pointed out, comparisons of aggregated performance between conditions in the Gar- ner paradigm mask certain confounds inherent within the original design. In particular, the effect of increasing the number of stimuli from two in the baseline condition to four in the filtering condition (i.e., stimulus uncertainty) and the contribution of two novel types of intertrial contingency in the filtering condition (i.e., irrelevant variation) cannot be separated on the basis of aggregate condition RT. When repetition effects were used to resolve this issue, both the presence of irrelevant variation and the increase in stimulus uncertainty had a minimal contribution when (faster) pitch was relevant and (slower) loudness was irrelevant, consistent with the lack of a significant overall filtering cost for pitch responding. In contrast, when (slower) loudness was relevant and (faster) pitch was irrelevant, an overall filtering cost for loudness responding was observed, with repetition effects providing for the first time independent estimates of the processing cost caused by the presence of irrelevant variation and the increase in stimulus uncertainty. The present data suggest that both effects contribute to overall filtering costs, 
Table 5

Results of the Paired-Sample $t$ Test Between Pairwise Attributes (Pitch, Loudness, Azimuth) and Against Zero, for the Estimates of Filtering Cost (Irrelevant Variation, Stimulus Uncertainty) and Correlated Benefit (Redundancy R, Redundancy C) Across Experiments 1-3

\begin{tabular}{|c|c|c|c|c|c|c|c|c|}
\hline & \multicolumn{4}{|c|}{ Filtering Costs } & \multicolumn{4}{|c|}{ Correlated Benefits } \\
\hline & \multicolumn{2}{|c|}{ Irrelevance } & \multicolumn{2}{|c|}{ Uncertainty } & \multicolumn{2}{|c|}{ Redundancy R } & \multicolumn{2}{|c|}{ Redundancy C } \\
\hline & $t$ & $p$ & $t$ & $p$ & $t$ & $p$ & $t$ & $p$ \\
\hline \multicolumn{9}{|c|}{ Experiment 1 (Pitch vs. Loudness) } \\
\hline Pitch vs. loudness & 4.39 & .001 & 3.46 & .004 & 1.10 & .291 & 0.97 & .347 \\
\hline Pitch against 0 & 2.42 & .029 & 0.49 & .634 & 2.55 & .022 & 1.98 & .067 \\
\hline Loudness against 0 & 5.32 & $<.001$ & 5.10 & $<.001$ & 5.65 & $<.001$ & 2.39 & .030 \\
\hline \multicolumn{9}{|c|}{ Experiment 2 (Azimuth vs. Loudness) } \\
\hline Azimuth vs. loudness & 0.14 & .889 & 3.38 & .004 & 3.87 & .001 & 3.96 & .001 \\
\hline Azimuth against 0 & 0.84 & .413 & 0.43 & .434 & 0.49 & .629 & 0.78 & .776 \\
\hline Loudness against 0 & 0.40 & .696 & 3.39 & .004 & 4.42 & $<.001$ & 4.83 & $<.001$ \\
\hline \multicolumn{9}{|c|}{ Experiment 3 (Azimuth vs. Pitch) } \\
\hline Azimuth vs. pitch & 1.62 & .126 & 0.11 & .912 & 1.13 & .277 & 0.52 & 611 \\
\hline Azimuth against 0 & 6.25 & $<.001$ & 2.47 & .026 & 0.06 & .952 & 0.91 & .378 \\
\hline Pitch against 0 & 3.31 & .005 & 3.09 & .007 & 1.32 & .206 & 0.01 & .992 \\
\hline
\end{tabular}

although the issue remains whether effects of stimulus uncertainty and irrelevant variation may themselves be dissociated from one another, thereby providing evidence for relatively early and late influences in processing interference as a result of filtering.

The interaction between pitch and loudness dimensions was further pursued by comparison between correlated and baseline conditions. In particular, two forms of redundancy (i.e., redundancy repetition and repetition change) were compared, with the aim of evaluating whether the identity of the task-irrelevant dimension influenced processing during correlated conditions. In contrast to the hypothesis that change trials should lead to greater differences between correlated and baseline conditions, relative to repeat trials, redundancy repetition was found to be the more robust measure. Therefore, although complete stimulus repetition in both correlated and baseline conditions represents the potential reuse of both dimensions (Felfoldy, 1974), the additional speeding in the correlated condition suggests that only in this condition do both features influence processing. This compromises the claim that dimensional interaction is inflexible (Dyson \& Quinlan, 2004): Task-irrelevant information that inhibits responding during filtering and facilitates responding during correlation can essentially be disregarded during baseline (similar arguments have been put forward regarding the presence of congruency effects in filtering and correlated conditions, but not in baseline conditions; see Patching \& Quinlan, 2002). In terms of revealing a preference for initiating response shortcuts on the basis of complete stimulus repetition and complete stimulus change (Pashler \& Baylis, 1991), the bypassing of central processing was initiated more quickly under the former contingency during correlated conditions. The idea that a primary goal of environmental analysis is to check for invariance across consecutive perceptual samples (e.g., Dyson, Alain, \& He, 2005 ) is a principle common to a number of models of performance related to same/different judgments (Bam- ber, 1969; Farell, 1985; Hawkins, 1969; Watanabe, 1988), structurally similar to the types of computation required in the present experiments (Dyson \& Quinlan, 2004; Entus \& Bindra, 1970).

Finally, the data also support the observation that although filtering costs and correlated benefits are used as converging evidence for certain types of stimulus dimension relationships (Grau \& Kemler Nelson, 1988), they may represent fundamentally different forms of processing, given that they can be dissociated from one another (cf. Boenke et al., 2009; Patching \& Quinlan, 2002; van Leeuwen \& Bakker, 1995). However, the data provide a warning in this regard, in that macrolevel assumptions regarding dissociation do not necessarily imply dissociation at a microlevel. For example, on the basis of the macrolevel analyses (i.e., comparisons involving condition averages), performance across the various pitch conditions was shown to be equivalent. However, an examination of intertrial contingencies revealed a significant contribution of redundancy during repetition in correlated conditions and showed that the absence of filtering costs was due to the summation of a positive (significant) contribution from stimulus irrelevance and a negative contribution from stimulus uncertainty. Experiment 1 demonstrated that microlevel analyses may be more sensitive than macrolevel analyses in providing evidence for integrality between stimulus dimensions.

\section{EXPERIMENT 2}

Although the data from Experiment 1 extend our understanding of Garner interference performance by emphasizing cross-trial relations, it is important to see how these previously hidden measures of task performance modulate as a function of using different auditory dimensions. Consequently, Experiment 2 examined the relationship between relatively fast azimuth and relatively slow loudness judgments under conditions identical to those in 
Experiment 1. Again on the basis of horse race models, an asymmetric separable relationship between azimuth and loudness should obtain at macrolevel (conditionaverage) performance, with both filtering costs and correlated benefits for loudness judgments, but not for azimuth judgments. Moreover, if microlevel (trial-to-trial) performance is completely predictable from macrolevel performance, the filtering cost should be represented by effects of both stimulus uncertainty and irrelevant variation, whereas the correlated benefit should be represented by effects of redundancy during repetition and change, as per Experiment 1.

The theoretical rationale for examining azimuth with loudness was that both attributes may be thought of as contributions to a multidimensional representation of acoustic space, just as putatively different acoustic properties such as attack time, spectral centroid, and spectrum fine analysis may be thought of as contributing to a multidimensional timbral space (e.g., Caclin et al., 2007; Caclin et al., 2008). Under the present design, azimuth may be thought of as representing variation in horizontal space, whereas loudness may be thought of as representing variation in distance. One reasonable suggestion, therefore, was that azimuth and loudness may show increased signs of perceptual integrality, since the localization of sound sources in 2-D space relies on both kinds of information.

\section{Method}

Participants. Informed consent was obtained for 16 participants consisting of 10 females and 13 right-handers, with ages ranging from 19 to 31 years $(M=24, S D=4.58)$.

Materials, Design, and Procedure. In Experiment 2, azimuth variation was defined relative to left and right monaural headphone presentation (with responses horizontally oriented), and loudness variation was again defined relative to soft (approximately $70 \mathrm{~dB}$ ) and loud (approximately $80 \mathrm{~dB}$ ) intensities carried by a 100 -msec, $1500-\mathrm{Hz}$ tone. All other parameters were identical to those in Experiment 1.

\section{Results}

Macrolevel analysis. RT data revealed main effects of dimension ( $p<.001)$, with azimuth judgments generally enacted more quickly than loudness judgments (see Tables 2 and 3$)$. The main effect of condition $(p<.001)$, together with an interaction between dimension and condition $(p<.001)$, revealed the absence of significant differences across baseline, filtering, and correlated conditions during azimuth judgment. In contrast, during loudness judgments, the filtering condition was significantly slower and the correlated condition was significantly faster than the baseline condition. Error rate analyses showed a similar interaction $(p=.010)$, in that, although there was no significant variation in error rates during azimuth judgments, error rates for loudness judgments were generally larger and particularly inflated under filtering conditions.

Microlevel analysis. Figure 1 provides a graphical representation of microlevel contributions to filtering costs and correlated benefits in Experiment 2 (see also Tables 4 and 5). In terms of filtering cost, irrelevant variation failed to depart from zero for azimuth ( $1 \mathrm{msec} ; p=$ .413 ) or loudness ( $1 \mathrm{msec} ; p=.696)$ responding, whereas an effect of stimulus uncertainty was present only during loudness responding ( $59 \mathrm{msec} ; p=.004)$. In terms of correlated benefits, neither redundancy during repetition ( $2 \mathrm{msec} ; p=.629$ ) nor redundancy during change ( $1 \mathrm{msec} ; p=.776$ ) was in evidence during azimuth responding, but both were present during loudness responding $(-25 \mathrm{msec}, p<.001$, and $-24 \mathrm{msec}, p<.001$, respectively). In terms of bypass initiation, preference for complete stimulus repetition (RR) over complete stimulus change (CC) was observed during loudness responding $[t(15)=3.31, p=.005]$ but not during azimuth responding $[t(15)=1.55, p=.141]$.

\section{Discussion}

When compared with the data from Experiment 1, the data from Experiment 2 suggest that standard analyses of Garner interference are inappropriate indices of dimensional interaction. Specifically, superficial similarity at a macrolevel of performance can mask subtle differences in processing that exist at the microlevels of performance. On the basis of average condition RT, performance between pitch and loudness in Experiment 1 and between azimuth and loudness in Experiment 2 appear equivalent. In both cases, performance is adequately characterized by an asymmetrical separable relationship predictable from horse race modeling: Responding on the basis of the faster of two dimensions can operate independently from processing of the slower of two dimensions, whereas responding on the basis of the slower of two dimensions results in facilitated performance during correlated conditions and inhibited performance during filtering conditions (see Table 2). On the basis of aggregate condition RTs, one would be led to the conclusion that pitch and loudness interact with one another in a way that is identical to that of azimuth and loudness.

However, various microlevel effects associated with filtering costs and correlated benefits tell quite a different tale. Specifically, the analysis of repetition effects in Experiment 2 showed that stimulus uncertainty was essentially the sole cause of the lengthening of RTs in the loudness filtering condition. This clearly contrasts with the loudness filtering condition in Experiment 1, in which both stimulus uncertainty and irrelevant variation appeared to contribute to the overall effect (see Figure 1). Therefore, it seems that when stimulus dimensions are at least asymmetrically integral, some lengthening of RTs as a consequence of increased stimulus uncertainty in filtering conditions is mandatory, whereas RT lengthening as a consequence of the presence of irrelevant variation is not. This is broadly consistent with the suggestion that stimulus uncertainty exerts an influence on processing earlier than does irrelevant variation, given the explicit retrieval of dimensional information required by the latter.

The idea of asymmetrical integrality was further supported by the observation of correlated benefits during loudness responding: Redundancy during repetition and redundancy during change were of an equivalent magnitude, and redundancy during repetition was significantly different from zero. Both data points suggest that the featural value of a secondary dimension exerts an influence 
on responding only when it varies across trials. Once again, this calls into question the absolute nature of dimensional integrality, if information on a task-irrelevant dimension (in this case, azimuth) can be abandoned during baseline performance. Consistent with Experiment 1, the bypassing of central processing as a result of complete repetition and change appeared to favor repetition (Hawkins, 1969; Pashler \& Baylis, 1991; Watanabe, 1988).

Therefore, despite surface similarity between the nature of dimensional interaction (i.e., asymmetrical integral) between pitch and loudness, on the one hand (Experiment 1), and azimuth and loudness, on the other (Experiment 2), the relative contributions of these effects, as indexed by intertrial contingency, are quite different. It is worth considering why only the effect of stimulus uncertainty was present for combinations of azimuth and loudness in Experiment 2 but the effects of both stimulus uncertainty and irrelevant variation were present for combinations of pitch and loudness in Experiment 1. It can be noted that the difference between the fast and slow dimensions was smaller in the case of pitch and loudness (Experiment 1), relative to the case of azimuth and loudness (Experiment 2). This rules out an account of dimensional interaction based on relative speeds of processing, since azimuth information would have been available earlier and, hence, had more time to influence loudness processing in Experiment 2. Alternatively, it may be the case that the expression of irrelevant variation requires stimulus dimensions to run closer in tandem with one another in terms of baseline discriminability, such that the potential degree of cross talk is increased (Pomerantz, 1983). Experiment 3 provided a final example of auditory dimensional interaction with which to evaluate these ideas.

\section{EXPERIMENT 3}

One particular set of auditory dimensions that have received a substantial amount of interest is the pairing of azimuth and pitch (e.g., Anourova et al., 2001; Dyson \& Quinlan, 2002, 2003, 2004; Leboe \& Mondor, 2007; Mondor \& Leboe, 2008; Rusconi, Kwan, Giordano, Umiltà, \& Butterworth, 2006; Schröger \& Wolff, 1997; Woods, Alain, Diaz, Rhodes, \& Ogawa, 2001; Zmigrod $\&$ Hommel, 2009). One of the reasons why combinations of azimuth and pitch are of interest is that these forms of processing may be represented by anatomically distinct pathways within the brain: a dorsal where (location) pathway running from the auditory cortex to the inferior frontal gyrus and a ventral what (identity) pathway running from the posterior temporal cortex to the superior frontal sulcus (e.g., Alain, Arnott, Hevenor, Graham, \& Grady, 2001; Arnott, Binns, Grady, \& Alain, 2004; although see Hall, 2003).

Consequently, there seems to be a priori anatomical evidence for arguing that azimuth and pitch processing should be separable to a certain degree, but behavioral data have been ambiguous in this regard. Support for some degree of what and where separability has come from a study reported by Näätänen, Porkka, Merisalo, and Ahtola (1980). In that case, participants were required to make go/no-go responses to relevant and irrelevant stimuli, respectively, with the relevant stimulus being defined by a combination of azimuth and pitch. It was found that the closer the irrelevant stimulus was in pitch to the relevant stimulus, the longer the RT, but this effect of pitch proximity was observed only when the irrelevant stimulus also shared location with the relevant stimulus. If the irrelevant stimuli did not share location, pitch proximity did not have an effect on the data. In this respect, Näätänen et al. reported a pattern of data similar to the asymmetric integral relationships discussed in Experiments 1 and 2.

In a series of behavioral studies reported by Mondor et al. (1998), integrality between pitch and azimuth information was observed using average condition performance across baseline and filtering condition similar to those presently employed. Despite azimuth responses being significantly faster than pitch responses, Mondor et al. (1998) observed filtering costs of similar magnitude, arguing for the symmetrical integrality between what and where systems in audition (see also Dyson \& Quinlan, 2004). Therefore, Experiment 3 examined azimuth and pitch combinations in a bid to examine how performance at the macrolevel may or may not mask more detailed effects at the microlevel. This experiment also provides a final test of the applicability of horse race accounts of processing to audition (Mordkoff \& Yantis, 1991; Mordkoff et al., 1990), which, so far, has been adequate in explaining the macrolevel relationships between pitch and loudness and between azimuth and loudness.

On the basis of our previous data in which azimuth was relatively fast (Experiment 2) and pitch was relatively slow (Experiment 1) under conditions of baseline discriminability (see Table 1), irrelevant azimuth should inhibit pitch responding in the filtering condition and/or facilitate pitch responding in the correlated condition, but pitch processing should not interfere with azimuth processing.

\section{Method}

Participants. The final sample of 16 participants had an age range from 19 to 42 years $(M=21.57, S D=5.68)$, with 14 females and 14 right-handers.

Materials, Design, and Procedure. To create the stimuli for Experiment 3, pitch variation from Experiment 1 was combined with azimuth variation from Experiment 2. In order to equate for subjective loudness across frequencies (Woods et al., 1998), low tones were calibrated to $76 \mathrm{~dB}$ and high tones to $74 \mathrm{~dB}$. All other parameters were identical to those in Experiments 1 and 2.

\section{Results}

Macrolevel analysis. RT data revealed main effects of dimension $(p=.004)$ and condition $(p<.001)$ in the absence of a significant interaction $(p=.768$; see Tables 2 and 3). Azimuth judgments (343 $\mathrm{msec})$ were revealed to be faster than pitch judgments $(397 \mathrm{msec})$, and responses in the filtering condition (397 $\mathrm{msec})$ were slower than responses in either the baseline or the correlated condition (357 and $356 \mathrm{msec}$, respectively). Error rates failed to show any significant main effects or interactions.

Microlevel analysis. Figure 1 provides a graphical representation of filtering cost and correlated benefit decomposition in Experiment 3 (see also Tables 4 and 5). 
With respect to the contributions of irrelevant variation and stimulus uncertainty on filtering costs, azimuth responding estimates did not differ from pitch responding estimates ( $p=.126$ and .912 , respectively), and all costs were significantly different from zero (14 and $8 \mathrm{msec}$ and 28 and $30 \mathrm{msec}$, respectively; all $p$ s $<.026)$. With respect to correlated benefits, no statistically significant effects of redundancy were observed (all $p \mathrm{~s}>$.206). As in all the previous experiments, bypass initiation seemed to favor complete stimulus repetition (RR), relative to complete stimulus change $(\mathrm{CC})$, which was statistically significant during pitch $[t(15)=3.21, p<.001]$ but not azimuth $[t(15)=1.94, p=.072]$ responding.

\section{Discussion}

The data from Experiment 3 examining the relationship between azimuth and pitch both replicate and depart from previous data sets in a number of important ways. Condition average RT supports the symmetrical integrality between azimuth and pitch, as reported by Mondor et al. (1998) and Dyson and Quinlan (2004), in revealing filtering costs for both dimensions despite differences in baseline discriminability (see Table 2). Thus, combinations of azimuth and pitch appear to represent a reliable violation of horse race principles (see also Dyson, in press, for similar violations involving elevation and pitch). Specifically, in Experiments 1 and 2, the slower dimension had no effect on the processing of the faster dimension, but in Experiment 3, the slower dimension (pitch) had an effect on the processing of the faster dimension (azimuth). At the very least, these data suggest that there are early levels of interaction between what and where processing that fail to be captured by choice RT (Patching \& Quinlan, 2002; Pomerantz, 1983). At the most, the data also suggest that pitch is indispensable in the processing of acoustic information, such that it cannot be disregarded even when it is both slow and irrelevant to the task in hand (cf. Kubovy \& Van Valkenburg, 2001, 2003, with Neuhoff, 2003).

An analysis of microlevel performance revealed that the processing inefficiency apparent in the filtering condition was due to effects of both stimulus uncertainty and irrelevant variation and that the magnitudes of these effects were essentially independent of dimension: Putatively faster and slower dimensions in Experiment 3 behaved in a manner similar to the slower dimension in Experiments 1 and 2 . The data, however, provide no indication that the nature of cross talk leading to the processing interference in filtering conditions is qualitatively different for azimuth and pitch.

One possibility, based on our discussion of Experiments 1 and 2, is that when the faster dimension is irrelevant, the likelihood of retrieving featural information prior to the retrieval of the slower task-relevant dimension is increased, enhancing any irrelevant variation effects, relative to when the irrelevant dimension is the slower of the two. However, estimates of irrelevance were equivalent between azimuth and pitch responding ( $p=.126)$ and numerically favored the irrelevant dimension when it was slower (pitch). In terms of performance in the correlated conditions, no statistically significant microlevel effects were found, but bypass once again appeared to be initiated more quickly for complete stimulus repetition than for complete stimulus change. Therefore, in contrast to Experiments 1 and 2, in which significant microlevel correlated benefits were in evidence whenever significant microlevel filtering costs were also in evidence (see Figure 1), Experiment 3 showed filtering costs in the absence of correlated benefits, questioning the extent to which these effects represent the same underlying construct of integrality between stimulus dimensions.

\section{CROSS-EXPERIMENT ANALYSES}

Before the broader implications of the data are discussed, questions remain regarding the extent to which specific dimensional relationships are due to the absolute or relative differences between speeds of processing or are due to the intrinsic nature of the dimensions themselves. Given the completion of the pairwise comparisons between pitch, loudness, and azimuth, it is now possible to address some of these queries. To take one comparison at a macrolevel of analysis, the relationship between pitch and loudness in Experiment 1 may be broadly described as asymmetrically integral, whereas the relationship between azimuth and pitch in Experiment 3 may be broadly described as symmetrically integral. When average baseline RTs were compared across Experiments 1 and 3 according to fast (pitch in Experiment 1, azimuth in Experiment 3) and slow (loudness in Experiment 1, pitch in Experiment 3) dimensions, the main effect of dimension $\left[F(1,30)=10.82, M S_{\mathrm{e}}=3,508, p=.003\right.$, $\left.\eta_{\mathrm{p}}^{2}=.265\right]$ confirmed that discrimination of one dimension was slower than the other, yet the absence of a main effect of experiment $\left[F(1,30)=1.84, M S_{\mathrm{e}}=3,508, p=\right.$ $\left..185, \eta_{\mathrm{p}}^{2}=.058\right]$ and the interaction between experiment and dimension $\left[F(1,30)=0.48, M S_{\mathrm{e}}=13,778, p=.492\right.$, $\left.\eta_{\mathrm{p}}^{2}=.016\right]$ supports the idea that neither absolute nor relative speeds of processing, respectively, differed between the two experiments. Therefore, neither relative nor absolute differences in baseline discrimination (Melara \& Mounts, 1993) fully predict the overall perceptual relationship observed between dimensions.

A final and important pattern of data is revealed by comparing baseline discrimination of the three auditory dimensions used here as a function of dimensional pairing across experiments (see Table 2). Specifically, baseline RTs to pitch did not differ as a function of loudness or azimuth $[t(30)=0.24, p=.815]$ and baseline RTs to loudness did not differ as a function of pitch or azimuth $[t(30)=-0.34, p=.734]$, but baseline RTs to azimuth were faster when paired with loudness, relative to pitch $[t(30)=2.89, p<.007]$. Although it initially seems nonsensical that discrimination in the absence of change in an irrelevant dimension should be influenced by what is held constant in baseline conditions, it should be noted that in the interests of counterbalancing in Experiment 3 (and indeed, within all the experiments), half of the participants completed azimuth judgments first and half completed azimuth judgments second. Therefore, one possibility is that the source of baseline interference may derive from 
the previous mapping from pitch for half the participants. The influence of counterbalancing was supported by a between-participants two-way ANOVA interaction between dimension pairing (azimuth paired with loudness, Experiment 2; azimuth paired with pitch, Experiment 3) and dimension order (azimuth first, azimuth second) $\left[F(1,28)=12.20, p=.001, \eta_{\mathrm{p}}^{2}=.303\right]$. Although azimuth judgments in Experiment 2 were unaffected by dimension order, the idea that interference from the previous mapping caused baseline slowing for azimuth judgments when paired with pitch was refuted by the observation that azimuth judgments in Experiment 3 were actually slower when completed first. These data are consistent with other empirical work (Dyson, in press, Experiment 2) in which response mapping does not provide an adequate explanation for the detrimental effect of pitch on azimuth. This is further testament to the fundamental level at which putatively slow pitch judgments disrupt the efficient processing of putatively fast conceptualizations of location (Dyson \& Quinlan, 2004; Mondor et al., 1998) and to the potentially indispensable nature of pitch in acoustic responding (Kubovy, 1981).

\section{GENERAL DISCUSSION}

Garner interference is considered to be a central measure with which to evaluate the nature of interaction between multidimensional stimuli, and particular interest has been given to the differences across three condition types (baseline, filtering, and correlated) in order to assess the degree of separability or integrality between the attributes of interest. The aim of the experimental series was to demonstrate the utility of repetition effects, relative to condition average measures, by titrating filtering costs, relative to baseline, into separate contributions of stimulus uncertainty and irrelevant variation and by titrating correlated benefits, relative to baseline, into separate contributions of redundancy during repetition and redundancy during change. These observations will serve as the focus for the remainder of the discussion.

First, less efficient processing in the filtering condition, relative to the baseline condition, can be considered one piece of evidence toward dimensional integrality: Participants should be sensitive to the orthogonal variation of relevant and irrelevant attributes during filtering conditions, relative to variation in the relevant attribute alone during baseline conditions. However, there are two possible loci of effects for less efficient processing in the filtering condition: disruption due to an increase in the number of stimuli and disruption due to the presence of irrelevant variation. By decomposing aggregate condition RTs into various cross-trial effects (see Estimators 2 and 3), it has been possible to decompose filtering costs into effects of stimulus uncertainty and irrelevant variation. Across Experiments 1-3, the rise in stimuli from two in baseline conditions to four in filtering conditions was a reliable contributor to the RT costs associated with filtering. In contrast, the magnitude of irrelevant variation failed to play a role in the filtering cost during the loudness responding observed in Experiment 2. Therefore, increases in stimulus uncertainty and the introduction of irrelevant variation provide independent contributions to the overall filtering cost. In contrast to the detection of irrelevant variation, sensitivity to an increase in stimulus uncertainty need not rely on the explicit representation of dimensional or featural information (Lockhead, 1972) and, as such, undermines the claim that filtering costs, in and of themselves, reflect the structural nature of stimulus representation. Although the increase in stimulus uncertainty and the presence of irrelevant variation are intimately connected in the standard Garner design, further designs could separate these effects to a greater degree. In particular, multiple stimulus tokens could be used to represent certain dimensional categories (e.g., Melara \& Mounts, 1994; Mondor, Hurlburt, \& Thorne, 2003), thereby addressing whether increasing stimulus uncertainty along the relevant dimension is equivalent in terms of processing disruption to increasing stimulus uncertainty along the irrelevant dimension.

Second, a closer examination of filtering costs revealed that similar forms of macrolevel performance did not reflect similar forms of microlevel performance. Specifically, the contrast between Experiments 1 and 2 revealed that asymmetrical integral relationships (Pomerantz \& Sager, 1975) suggested by condition average performance can be expressed in quite different ways: For pitch and loudness (Experiment 1), both irrelevant variation and stimulus uncertainty contributed to the filtering cost; for azimuth and loudness (Experiment 2), only stimulus uncertainty appeared to slow responding, relative to the baseline condition. One suggestion is that, in both experiments, both auditory dimensions were processed on each trial during the slower filtering condition (cf. Duncan \& Humphreys, 1989; Mondor et al., 1998) but that additional time was then required to suppress the irrelevant dimension. Given the seemingly larger magnitude of difference in speeds of processing between azimuth and loudness, relative to pitch and loudness, this additional stage becomes possible in the former case, but not in the latter. This proposal could be tested by varying the relative speeds of processing for the same pairs of dimensions (Huetell \& Lockhead, 1999). For the moment, the data demonstrate that at least part of the filtering cost was simply associated with increasing the stimuli from two to four (an effect not necessarily associated with integrality per se) and that, in the case of Experiment 2, participants were sensitive to an increase in the number of stimuli within a given condition without necessarily showing sensitivity to the irrelevant features of those stimuli.

Third, more efficient processing in the correlated condition, relative to the baseline condition, is also considered to be another piece of evidence supporting dimensional integrality: Participants should be sensitive to the synchronized variation of relevant and irrelevant attributes during correlated conditions, relative to variation in the relevant attribute alone during baseline conditions. The analysis of repetition effects has also been useful in assessing additional constraints in these conditions (see Estimators 6 and 7). Specifically, the data suggested that a precondition of observing evidence for integrality was variation in both 
the relevant and irrelevant dimensions within a block of trials. This was first supported by the observation that correlated benefits were observed in intertrial contingencies involving complete stimulus repetition (RR trials) between correlated and baseline conditions. This presents the possibility that since the nature of stimulus and response transitions were identical, this effect of redundancy during repetition (redundancy $R$ ) may have been due to differences in stimulus representation, whereby both dimensional values influenced responding during correlated conditions but irrelevant dimensional values could be ignored during baseline conditions. Similarly, there was no indication that the effects of redundancy during change (redundancy $C$ ) were more robust than the effects of redundancy $\mathrm{R}$, which might have been expected if both dimensional values influenced responding during baseline conditions. The data call into question the absolute nature of dimensional interaction and further highlight the need to identify the environmental conditions under which the same stimulus dimensions can reflect integral or separable representation to differing degrees (see Dyson, in press; Dyson \& Quinlan, 2004).

Fourth, Estimator 9 provided a modest contribution to the issue of whether the default position of our processing system is to detect change or repetition by comparing both expressions of the bypass rule (Kruger \& Shapiro, 1981; Pashler \& Baylis, 1991; Rabbitt, 1992). The rule states that when both stimulus and response are either completely repeated (RR trials) or completely changed (CC trials), central processing may be ignored in favor of speeded responding. Across Experiments 1-3, when the comparison of RR and CC trials was statistically reliable, it always favored complete stimulus repetition, rather than complete stimulus change. Much has been made, at least regarding the auditory system, of the putative automaticity of change detection as indexed by such electrophysiological measures as mismatch negativity, in which change in a standard paradigm is the exception to the rule of repetitiously presented stimuli (e.g., Sabri \& Campbell, 2001; for reviews, see Garrido, Kilner, Stephan, \& Friston, 2009; Näätänen \& Winkler, 1999). Recently, however, consideration has also been given to the processing benefits accrued when repetition is the exception to the rule of continuously changing stimuli (Dyson \& Alain, 2008; Haenschel, Vernon, Dwivedi, Gruzelier, \& Baldeweg, 2005). Although, clearly, the importance attached to the detection of repetition and change will vary as a function of the statistical regularities of the environment, the present data provide evidence that when the probabilities of repetition and change are equivalent, we are quicker to respond to repetitious input. The decomposition of condition performance into intertrial contingency also helps to identify two further estimators that might be used in future experiments, in which the magnitude of bypass (and hence, the degree of integrality) between correlated and filtering condition may be compared by

$$
\{\mathrm{E}(\mathrm{RT} \mid \mathrm{RR} \text {; filtering })-\mathrm{E}(\mathrm{RT} \mid \mathrm{RR} \text {; correlated })\}
$$

and

$$
\{\mathrm{E}(\mathrm{RT} \mid \mathrm{CC} \text {; filtering })-\mathrm{E}(\mathrm{RT} \mid \mathrm{CC} \text {; correlated })\} .
$$

Fifth, the purely auditory data of the present series reveal that, like purely visual (e.g., Boenke et al., 2009) or bimodal (e.g., Patching \& Quinlan, 2002) processing, filtering costs and correlated benefits are dissociable from one another and, as such, may represent subtly different forms of evidence for dimensional integrality. In particular, Experiment 1 provided some evidence that (slower) loudness does not produce a filtering cost for (faster) pitch but can produce a correlated benefit (see also Patching \& Quinlan, 2002; van Leeuwen \& Bakker, 1995), and Experiment 3 provided some evidence that both (slower) pitch and (faster) azimuth provide mutual filtering costs but neither provides significant correlated benefits. Boenke et al. accounted for the distinction between these two forms of evidence by arguing that filtering costs represent between-trial interference and correlated benefits represent within-trial interference (see also Felfoldy, 1974). The present analyses speak to the question of the extent to which this is an accurate description of correlated and filtering effects.

Although the present analyses predominately emphasized between-trial (e.g., repetition) performance, the estimators presented in the introduction may represent two subtly different forms of effect. In terms of filtering costs, it could be argued that whereas Estimator 2 (irrelevant variation) represents a pure estimate of certain betweentrial effects, given that calculations are confined to a single condition, Estimator 3 (stimulus uncertainty) represents between-trial effects mixed with within-trial effects, given that the same intertrial relations are compared across conditions. Therefore, irrelevant variation may reflect the contribution of between-trial effects on filtering costs, but stimulus uncertainty may reflect within-trial effects as a result of the potentially different forms of stimulus representation employed across conditions. Consequently, the data reveal that there is a contribution to filtering costs (i.e., stimulus uncertainty) that may not be purely derived from between-trial effects and also hint that further estimators may be developed that identify contributions to correlated benefits that may not be purely derived from within-trial effects.

Alternative accounts of the data do, however, suggest themselves. One contribution that requires some consideration is the influence of stimulus-response (S-R) compatibility (Kornblum, Hasbroucq, \& Osman, 1990). Previous research has detailed complex interactions between dimension and task, such as incongruent location values slowing responding relative to other dimensions, except when the value of location is held constant across a block of trials (e.g., Wühr, Biebl, \& Ansorge, 2008). Within the present series, however, all responses were mapped so as to preserve the dominant spatial stereotype associated with each dimension (i.e., pitch, high and low; loudness, high and low; azimuth, left and right), thereby limiting the claim that S-R effects were exaggerated for one particular dimension. Nevertheless, it could be argued that this accounts for any filtering costs observed when location was irrelevant, given that, in baseline conditions, there was no irrelevant variation, whereas in filtering conditions, irrelevant variation was apparent (the same account 
is not easily applied to processing facilitation, as in the case of correlated benefits). However, there is nothing in the experimental series to suggest that irrelevant location interfered more, relative to pitch or loudness, during filtering conditions, as would be predicted by this account. Figure 1 clearly shows that in terms of numerical size, irrelevant pitch on loudness yielded the largest overall filtering cost in Experiment 1. Other concerns may be raised by the use of both horizontal and vertical response orientations across the experiments. For example, the absence of an irrelevant variation effect for loudness filtering in Experiment 2 may be explained in terms of such effects existing at a response-mapping level. In Experiment 1, both pitch and loudness responses were mapped vertically. In Experiment 2, pitch responses were mapped vertically, whereas azimuth responses were mapped horizontally. Therefore, there may have been less interaction between azimuth and loudness dimensions as a result of the different response orientation (despite the fact that $A_{0}$ and $\mathrm{B}_{0}$, and $\mathrm{A}_{1}$ and $\mathrm{B}_{1}$, were physically mapped onto the same hand in all the experiments). Such an account is refuted by Experiment 3, which provides evidence for irrelevant variation using a horizontal response mapping for azimuth and a vertical response mapping for pitch. Furthermore, arguments relating to differences in S-R compatibility rely on between-condition, rather than within-condition, comparisons. This suggests that the between-condition filtering estimator relating to stimulus uncertainty (3) may be more susceptible to S-R compatibility issues than the within-condition filtering estimator relating to irrelevant variation (2). Yet, across the experimental series, it was irrelevant variation, not stimulus uncertainty, that was the least reliable measure (see Experiment 2) and, as such, requires an explanation distinct from S-R mappings.

Issues surrounding $\mathrm{S}-\mathrm{R}$ compatibility more generally raise the consideration of congruency effects, with a distinction being made between S-R congruency (described above) and stimulus-stimulus congruency (e.g., Patching \& Quinlan, 2002). The latter type of relationship is most clearly expressed by examining the two versions of the correlated conditions (see the Method section of Experiment 1). Despite the value of each dimension being predictive of the other in both cases, during congruent correlated conditions, access to either dimension provides convergent mapping (e.g., $\mathrm{A}_{0} \mathrm{~B}_{0}, \mathrm{~A}_{1} \mathrm{~B}_{1}$ ), whereas during incongruent correlated conditions, access to either dimension provides divergent mapping (e.g., $\mathrm{A}_{1} \mathrm{~B}_{0}, \mathrm{~A}_{0} \mathrm{~B}_{1}$ ). Although analyses of stimulus token effects are informative for a number of reasons (e.g., evaluating a stimulus-driven account of conflict adaptation; Mayr, Awh, \& Laurey, 2003), there are reasons why stimulus type transitions (i.e., intertrial contingencies) and stimulus token (i.e., congruency) transitions should be considered independently from one another. For example, in Estimator 7, performance is compared across CC correlated trials and CR baseline trials to establish redundancy during change (redundancy C). By definition, $\mathrm{CC}$ contingencies in correlated conditions must constitute either congruent-congruent or incongruent-incongruent stimulus token transitions, whereas CR contingencies in baseline conditions must constitute either congruent-incongruent or incongruentcongruent stimulus token transitions. Consequently, there is an extent to which it is not possible to examine stimulus type effects while examining stimulus token effects, and one would need to collapse over intertrial contingency to do so. Additional valid comparisons, however, do suggest themselves, and in particular, it should be possible to decompose the intertrial contingencies found in the filtering condition (RR, RC, CR, CC) according to whether participants also move to and from congruent or incongruent stimuli across trials (cf. Mayr et al., 2003). Although congruency effects are apparent in some of our data, we are currently considering the implications of examining the data concurrently with respect to both stimulus type and stimulus token transitions in this super-microlevel of analysis. Although both S-R compatibility and stimulus congruency effects suggest further levels of decomposition, we have controlled for these effects as well as the present design could allow by having all valid transitions between stimulus tokens and all valid S-R compatibility relationships appear with equal probability within the respective conditions. We believe that despite these pending issues, the present data provide a useful intermediate step in highlighting the relationship between low- and highfidelity levels of analysis in terms of performance during Garner interference.

In sum, the data provide further evidence for intertrial contingencies providing insights into processing constraints that ordinarily would not be revealed by simple condition-average analyses. The appeal for repetition effects' playing a central role in the analysis of experimental data does not necessitate significant time or resource investments: With appropriate stimulus coding, it is possible to retrieve intertrial contingency data at a later date from preexisting and future data sets. Taking the time to review what happens on a trial-by-trial basis not only highlights deeper processing structures within traditional psychological paradigms, but also acknowledges the fundamental role that repetition plays in modulating electrophysiological and hemodynamic responses to our own external world in the pursuit of efficient sensory coding (Baldeweg, 2006; Grill-Spector, Henson, \& Martin, 2006; Smith \& Lewicki, 2006). Curtailing processing for aspects of our environment that remain the same and deploying processing for aspects of our environment that change is one way in which sensitivity to moment-to-moment relations can assist in offsetting the substantial demands of perceptual analysis. A large part of understanding our own efficiency is to appreciate how our processing system is constantly fine-tuned to the amount of repetition and change within our environment, demonstrating maximal sensitivity to old and new experiences alike.

\section{AUTHOR NOTE}

B.J.D. is supported by an Early Researcher Award granted by Ontario Ministry of Research and Innovation. Some of the data were presented at the 46th Annual Meeting of the Psychonomic Society, November 10-13, 2005, Toronto. The authors thank Stephen Want for helpful discussion during manuscript preparation, and Simon Grondin, Anne Caclin, and an anonymous individual for constructive comments during the review process. Correspondence should be addressed to B. J. Dyson, Department of 
Psychology, Ryerson University, 350 Victoria Street, Toronto, ON, M5B 2K3 Canada (e-mail: ben.dyson@psych.ryerson.ca).

\section{REFERENCES}

Alain, C., Arnott, S. R., Hevenor, S., Graham, S., \& Grady, C. L. (2001). "What" and "where" in the human auditory system. Proceedings of the National Academy of Sciences, 98, 12301-12306.

Anourova, I., Nikouline, V. V., Ilmoniemi, R. J., Hotta, J., Aronen, H. J., \& CARLSON, S. (2001). Evidence for dissociation of spatial and nonspatial auditory information processing. NeuroImage, 14, 12681277.

Arnott, S. R., Binns, M. A., Grady, C. L., \& Alain, C. (2004). Assessing the auditory dual-pathway model in humans. NeuroImage, 22, 401-408.

BALDEWEG, T. (2006). Repetition effects to sounds: Evidence for predictive coding in the auditory system. Trends in Cognitive Sciences, 10, 93-94.

BAMBER, D. (1969). Reaction times and error rates for "same""different" judgments of multidimensional stimuli. Perception \& Psychophysics, 6, 169-174.

Ben-Artzi, E., \& Marks, L. E. (1995). Visual-auditory interaction in speeded classification: Role of stimulus difference. Perception \& Psychophysics, 57, 1151-1162.

Ben-Artzi, E., \& Marks, L. E. (1999). Processing linguistic and perceptual dimensions of speech: Interactions in speeded classification. Journal of Experimental Psychology: Human Perception \& Performance, 25, 579-595.

Boenke, L. T., Ohl, F. W., Nikolaev, A. R., Lachmann, T., \& van Leeuwen, C. (2009). Different time courses of Stroop and Garner effects in perception-An event-related potentials study. NeuroImage, 45, 1272-1288.

Caclin, A., Giard, M. H., Smith, B. K., \& McAdams, S. (2007). Interactive processing of timbre dimensions: A Garner interference study. Brain Research, 1138, 159-170.

Caclin, A., McAdams, S., Smith, B. K., \& Giard, M. H. (2008). Interactive processing of timbre dimensions: An exploration with eventrelated potentials. Journal of Cognitive Neuroscience, 20, 49-64.

Cohen, J., MacWhinney, B., Flatt, M., \& Provost, J. (1993). PsyScope: An interactive graphic system for designing and controlling experiments in the psychology laboratory using Macintosh computers. Behavior Research Methods, Instruments, \& Computers, 25, 257-271.

Duncan, J., \& Humphreys, G. W. (1989). Visual search and stimulus similarity. Psychological Review, 96, 433-458.

Dyson, B. J. (in press). Trial after trial: General processing consequences as a function of repetition and change in multi-dimensional sound. Quarterly Journal of Experimental Psychology.

Dyson, B. J., \& Alain, C. (2008). Is a change as good with a rest? Task-dependent effects of inter-trial contingency on concurrent sound segregation. Brain Research, 1189, 135-144.

Dyson, B. J., Alain, C., \& He, Y. (2005). I've heard it all before: Perceptual invariance represented by early cortical auditory-evoked responses. Cognitive Brain Research, 23, 457-460.

Dyson, B. J., \& Quinlan, P. T. (2002). Within- and between-dimensional processing in the auditory modality. Journal of Experimental Psychology: Human Perception \& Performance, 28, 1483-1498.

Dyson, B. J., \& QuinLan, P. T. (2003). Feature and conjunction processing in the auditory modality. Perception \& Psychophysics, 65, 254-272.

Dyson, B. J., \& Quinlan, P. T. (2004). Stimulus processing constraints in audition. Journal of Experimental Psychology: Human Perception \& Performance, 30, 1117-1131.

Entus, A., \& BindRA, D. (1970). Common features of the "repetition" and "same-different" effect in reaction time experiments. Perception \& Psychophysics, 7, 143-148.

FARELL, B. (1985). "Same"-“different" judgments: A review of current controversies in perceptual comparisons. Psychological Bulletin, 98, 419-456.

Felfoldy, G. L. (1974). Repetition effects in choice reaction time to multidimensional stimuli. Perception \& Psychophysics, 15, 453-459.

Garner, W. R. (1974). The processing of information and structure. Potomac, MD: Erlbaum.

GARNER, W. R. (1976). Interaction of stimulus dimensions in concept and choice processes. Cognitive Psychology, 8, 98-123.
Garner, W. R., \& Felfoldy, G. L. (1970). Integrality of stimulus dimensions in various types of information processing. Cognitive Psychology, 1, 225-241.

Garrido, M. I., Kilner, J. M., Stephan, K. E., \& Friston, K. J. (2009). The mismatch negativity: A review of underlying mechanisms. Clinical Neurophysiology, 120, 453-463.

Grau, J. W., \& Kemler Nelson, D. G. (1988). The distinction between integral and separable dimensions: Evidence for the integrality of pitch and loudness. Journal of Experimental Psychology: General, 117, 347-370.

Grill-Spector, K., Henson, R., \& Martin, A. (2006). Repetition and the brain: Neural models of stimulus-specific effects. Trends in Cognitive Sciences, 10, 14-23.

Haenschel, C., Vernon, D. J., Dwivedi, P., Gruzelier, J. H., \& BALDEWEG, T. (2005). Event-related brain potential correlates of human auditory sensory memory-trace formation. Journal of Neuroscience, 25, 10494-10501.

Hall, D. A. (2003). Auditory pathways: Are "what" and "where" appropriate? Current Biology, 13, R406-R408.

Harris, J. D., Pikler, A. G., Hoffman, H. S., \& Ehmer, R. H. (1958). The interaction of pitch and loudness discriminations. Journal of Experimental Psychology, 56, 232-238.

HaWkins, H. L. (1969). Parallel processing in complex visual discrimination. Perception \& Psychophysics, 5, 56-64.

Huettel, S. A., \& Lockhead, G. R. (1999). Range effects of an irrelevant dimension on classification. Perception \& Psychophysics, 61, 1624-1645.

JoLICEUR, P. (1999). Concurrent response-selection demands modulate the attentional blink. Journal of Experimental Psychology: Human Perception \& Performance, 25, 1097-1113.

Kemler Nelson, D. G. (1993). Processing integral dimensions: The whole view. Journal of Experimental Psychology: Human Perception \& Performance, 19, 1105-1113.

Kornblum, S., Hasbrouce, T., \& Osman, A. (1990). Dimensional overlap: Cognitive basis for stimulus-response compatibility-A model and taxonomy. Psychological Review, 97, 253-270.

Kruger, L. E., \& Shapiro, R. G. (1981). Intertrial effects of samedifferent judgements. Quarterly Journal of Experimental Psychology, 33A, 241-265.

Krumhansl, C. L., \& Iverson, P. (1992). Perceptual interactions between musical pitch and timbre. Journal of Experimental Psychology: Human Perception \& Performance, 18, 739-751.

Kubovy, M. (1981). Concurrent-pitch segregation and the theory of indispensable attributes. In M. Kubovy \& J. R. Pomerantz (Eds.), Perceptual organization (pp. 55-98). Hillsdale, NJ: Erlbaum.

Kubovy, M., \& VAn Valkenburg, D. (2001). Auditory and visual objects. Cognition, 80, 97-126.

Kubovy, M., \& Van Valkenburg, D. (2003). In defense of the theory of indispensable attributes. Cognition, 87, 225-233.

Leboe, L. C., \& Mondor, T. A. (2007). Item-specific congruency effects in nonverbal auditory Stroop. Psychological Research, 71, 568-575.

Lockhead, G. R. (1972). Processing dimensional stimuli: A note. Psychological Review, 79, 410-419.

Maddox, W. T. (1992). Perceptual and decisional separability. In F. G. Ashby (Ed.), Multidimensional models of perception and cognition (pp. 147-180). Hillsdale, NJ: Erlbaum.

Mayr, U., Awh, E., \& Laurey, P. (2003). Conflict adaptation effects in the absence of executive control. Nature Neuroscience, 6, 450-452.

Melara, R. D., \& Marks, L. E. (1990). Interaction among auditory dimensions: Timbre, pitch, and loudness. Perception \& Psychophysics, 48, 169-178

Melara, R. D., \& MounTs, J. R. W. (1993). Selective attention to Stroop dimensions: Effects of baseline discriminability, response mode, and practice. Memory \& Cognition, 21, 627-645.

Melara, R. D., \& MounTs, J. R. W. (1994). Contextual influences on interactive processing: Effects of discriminability, quantity, and uncertainty. Perception \& Psychophysics, 56, 73-90.

Mondor, T. A., Hurlburt, J., \& Thorne, L. (2003). Categorizing sounds by pitch: Effects of stimulus similarity and response repetition. Perception \& Psychophysics, 65, 107-114.

Mondor, T. A., \& LeboE, L. C. (2008). Stimulus and response repetition effects in the detection of sounds: Evidence of obligatory retrieval and use of a prior event. Psychological Research, 72, 183-191. 
Mondor, T. A., Zatorre, R. J., \& Terrio, N. A. (1998). Constraints on the selection of auditory information. Journal of Experimental Psychology: Human Perception \& Performance, 24, 66-79.

Mordkoff, J. T., \& Yantis, S. (1991). An interactive race model of divided attention. Journal of Experimental Psychology: Human Perception \& Performance, 17, 520-538.

Mordkoff, J. T., Yantis, S., \& Egeth, H. E. (1990). Detecting conjunctions of color and form in parallel. Perception \& Psychophysics, 48, 157-168.

NÄ̈̈tÄnen, R., PorkKa, R., Merisalo, A., \& Ahtola, S. (1980). Location vs. frequency of pure tones as a basis of fast discrimination. Acta Psychologica, 44, 31-40.

NÄÄTÄNEN, R., \& WINKLER, I. (1999). The concept of auditory stimulus representation in cognitive neuroscience. Psychological Bulletin, 125, 826-859.

Neuhoff, J. G. (2003). Pitch variation is unnecessary (and sometimes insufficient) for the formation of auditory objects. Cognition, 87, 219224.

Pashler, H., \& Baylis, G. (1991). Procedural learning: 2. Intertrial repetition effects in speeded-choice tasks. Journal of Experimental Psychology: Learning, Memory, \& Cognition, 17, 33-48.

Patching, G. R., \& Quinlan, P. T. (2002). Garner and congruency effects in the speeded classification of bimodal signals. Journal of Experimental Psychology: Human Perception \& Performance, 28, $755-775$.

Pomerantz, J. R. (1983). Global and local precedence: Selective attention in form and motion perception. Journal of Experimental Psychology: General, 112, 516-540.

Pomerantz, J. R., \& SAger, L. C. (1975). Asymmetric integrality with dimensions of visual pattern. Perception \& Psychophysics, 18, 460466.

Quinlan, P. T. (1999). Sequential effects in auditory choice reaction time tasks. Psychonomic Bulletin \& Review, 6, 297-303.

RabBitT, P. (1992). Many happy repetitions: A celebration of the "Bertelson Repetition Effect” 1961-1991. In J. Alegria, D. Holender, J. Junca de Morais, \& M. Radeau (Eds.), Analytic approaches to human cognition (pp. 313-330). Amsterdam: Elsevier.

Rusconi, E., Kwan, B., Giordano, B. L., Umiltà, C., \& ButterWORTH, B. (2006). Spatial representation of pitch height: The SMARC effect. Cognition, 99, 113-129.

SABri, M., \& CAMPBell, K. B. (2001). Effects of sequential and temporal probability of deviant occurrence on mismatch negativity. Cognitive Brain Research, 12, 171-180.
Schröger, E., \& WolfF, C. (1997). Fast preattentive processing of location: A functional basis for selective listening in humans. Neuroscience Letters, 232, 5-8.

Shechter, S., \& Hochstein, S. (1992). Asymmetric interactions in the processing of the visual dimensions of position, width, and contrast of bar stimuli. Perception, 21, 297-312.

Sмith, E. C., \& LEWICKI, M. S. (2006). Efficient auditory coding. $\mathrm{Na}$ ture, 439, 978-982.

Treisman, A. M., \& Gelade, G. (1980). A feature-integration theory of attention. Cognitive Psychology, 12, 97-136.

VAN LEEUWEN, C., \& BAKKER, L. (1995). Stroop can occur without Garner interference: Strategic and mandatory influences in multidimensional stimuli. Perception \& Psychophysics, 57, 379-393.

VAN Selst, M., \& Jolicceur, P. (1994). A solution to the effect of sample size on outlier elimination. Quarterly Journal of Experimental Psychology, 47A, 631-650.

WATANABE, T. (1988). Effect of irrelevant differences as a function of the relations between relevant and irrelevant dimensions in the samedifferent task. Journal of Experimental Psychology: Human Perception \& Performance, 14, 132-142.

Winer, B. J. (1962). Statistical principles in experimental design. New York: McGraw-Hill.

Wood, C. C. (1975). Auditory and phonetic levels of processing in speech perception: Neurophysiological and information-processing analyses. Journal of Experimental Psychology: Human Perception \& Performance, 1, 3-20.

Woods, D. L., Alain, C., Diaz, R., Rhodes, D., \& Ogawa, K. H. (2001). Location and frequency cues in auditory selective attention. Journal of Experimental Psychology: Human Perception \& Performance, 27, 65-74.

Woods, D. L., Alain, C., \& OGawa, K. H. (1998). Conjoining auditory and visual features during high-rate serial presentation: Processing and conjoining two features can be faster than processing one. Perception \& Psychophysics, 60, 239-249.

WÜHr, P., BIEBL, R., \& Ansorge, U. (2008). The impact of stimulus and response variability on S-R correspondence effects. Journal of Experimental Psychology: Learning, Memory, \& Cognition, 34, 533-545.

Zmigrod, S., \& Hommel, B. (2009). Auditory event files: Integrating auditory perception and action planning. Attention, Perception, \& Psychophysics, 71, 352-362.

(Manuscript received August 4, 2009; revision accepted for publication April 10, 2010.) 